\title{
Industria 4.0 na auto-organização dos sistemas \\ produtivos
}

João Alvarez Peixoto

Luisa Muller Pereira
Laboratório de Automação Industrial. Unidade de Porto Alegre, Universidade Estadual do Rio Grande do Sul, Porto Alegre, RS, Brasil

E-mails: joao-peixoto@uergs.edu.br luisa-pereira@uergs.edu.br

Recebido em: 4 maio 2018. Aceito: 21 junho 2018.

DOI: http://dx.doi.org/10.21674/2448-0479.43.525-538

\section{Resumo}

Os requisitos de manufatura industrial apontam uma necessidade de reconfiguração e reprogramação do fluxo de processo, a fim de atender modificações no produto com as mudanças dos requisitos de mercado. Estas mudanças implicam em alterações no processo de fabricação, o que, em muitos casos, significa alterar o leiaute, reprogramar controladores, modificar acoplamentos e interfaceamentos, entre outros. As premissas da Indústria 4.0 trazem conceitos de modularização, orientação a serviços e adaptação em tempo real, o que dá ao processo produtivo as características de auto-organização, concedendo mais autonomia, para se autogerenciar e que permitam uma troca rápida de funcionalidades passam a serem desejados em um ambiente de manufatura, onde os sistemas convencionais com programação centralizada, sequência definida no controlador central e arranjo de funcionalidades fixas não dão conta das novas demandas fabris. Os equipamentos passam a se comportar como agentes de manufatura, num universo de multiagentes, que negociam entre si o processo requerido, propiciando o atendimento à variação de produto e dispondo de um número maior de funcionalidades, o que reduz o tempo de troca de processos. Um ensaio com sistemas de manufatura CIM, MES e auto-organizável é realizado, comparando seus resultados, frente a métricas que atendam a necessidade de produtos diversificados em um meio produtivo, onde os sistemas autoorganizáveis mostram seu valor aos novos requisitos de mercado. A auto-organização se apresentou como o sistema mais adequado para atender a requisitos de diversidade e customização de produtos.

Palavras-chave: Auto-organização. Multiagente. Indústria 4.0. Manufatura. Produção. 


\section{Abstract \\ Industry 4.0 in the self-organization of productive systems}

Industrial manufacturing requirements point to a need for reconfiguration and reprogramming of the process flow in order to meet product modifications with changes in market requirements. These changes imply changes in the manufacturing process, which in many cases means altering the layout, reprogramming controllers, modifying couplings and interfacing, among others. The premise of Industry 4.0 brings concepts of modularization, service orientation and adaptation in real time, which gives the productive process the characteristics of self-organization, granting more autonomy, to self-manage and allowing a quick change of functionalities become desired in a manufacturing environment, where conventional systems with centralized programming, a sequence defined in the central controller, and an array of fixed functionalities are in short supply. Equipment is to behave like manufacturing agents, a multi-agent universe, who trade with each other the required process, providing the service to the product variation and having a greater number of features, which reduces the time to exchange processes. An assay with CIM, MES and self-organizing manufacturing systems is performed by comparing its results against metrics that meet the need for diversified products in a productive environment where self-organizing systems show their value to new market requirements.

Keywords: Self-organization. Mult-agent. Industry 4.0. Manufacturing. Production.

\section{Introdução}

A indústria de manufatura tem se deparado com uma necessidade de mercado que remete ao consumo de produtos altamente personalizados. Durante vários anos o conceito de produção em massa, caracterizada pela produção do mesmo produto em grande escala, foi amplamente utilizado. Hoje em dia este conceito é incapaz de tratar as variações no tipo de produto, e não consegue mais responder aos desafios de modernidade e dinamismo. Grandes lotes de produção, linhas de produção com máquinas e processos idênticos e a padronização de produtos tendem a deixar de existir. A produção em grandes quantidades continua a ser demandada, porém, como aponta (MENDES et al., 2008), há uma tendência à produção em massa de produtos altamente personalizados. Qualidade e funcionalidade deixaram de ser os únicos atributos de um produto. A eles incorporaram-se diversidade, atualização, possibilidade de agregar funcionalidades, dentre outros. Atributos que remetem a um sistema de produção cada vez mais ágil, flexível e eficaz.

Preocupados com as mesmas demandas, o governo alemão lançou em 2011 na Feira de Hannover (Alemanha), um novo conceito como parte da estratégia para o desenvolvimento de alta tecnologia para a manufatura do país (EUROPEAN PARLIAMENT, 2015). O termo Indústria 4.0, também conhecida como a 4⿳亠丷厂 Revolução Industrial, vem sendo disseminado mundialmente e é marcada pelo conjunto de mudanças nos processos de manufatura, design, produto, operações e sistemas relacionados à produção, aumentando o valor na cadeia organizacional e em todo o ciclo de 
vida do produto. O 4.0 deriva da quarta versão, onde os mundos virtuais e físicos se fundem ao redor da planta operacional (fornecedores, distribuidores, unidades fabris, e até o produto), onde todos são conectados digitalmente, proporcionando uma cadeia de valor altamente integrada.

Segundo o estudo alemão da Technische Universitat Dortmund (HERMANN; PENTEK; OTTO, 2015), foram identificados seis requisitos para a implementação da Indústria 4.0:

a) Interoperabilidade, permitindo que todos os CPS de uma fábrica ou ambiente industrial, mesmo que descendentes de diversos fornecedores, possam se comunicar através das redes;

b) Virtualização, possibilitando que os dados obtidos dos CPS (Ciber Phisical Systems - Sistemas parte físico e parte virtual) nos produtos e equipamentos físicos sejam transmitidos aos modelos virtuais e em simulações, espelhando comportamentos reais no ambiente virtual;

c) Descentralização dos controles dos processos produtivos, uma vez que os computadores embarcados em conjunto com a internet das coisas gerarão produtos com tomadas de decisões na manufatura e nos processos de produção em tempo real;

d) Adaptação da produção em tempo real, uma vez que os dados serão analisados no instante em que são coletados, permitindo que a produção seja alterada ou transferida para outros silos em caso de falhas ou na produção de bens customizados;

e) Orientação a serviços, onde dados e serviços serão disponibilizados em rede aberta, onde a customização de processos de produção e operação terá maior flexibilidade de adaptação de acordo com as especificações dos clientes.

f) Sistemas modulares dos equipamentos e linhas de produção, tornando as fábricas mais flexíveis e adaptáveis às alterações necessárias.

Partindo destas premissas, a implementação de sistemas modulares, a descentralização dos controles dos processos e a orientação a serviços, remetem a um sistema auto-organizável, que permite ao fluxo de processos fabril se modificar de forma dinâmica dentro do meio fabril, com maior interatividade entre os elementos que compõe a produção.

O planejamento de recursos e fluxo produtivo necessitam serem constantemente alterados no sistema produtivo, a fim de atender as variações de produtos que são demandados pelo mercado. Mas a forma com que são planejados os fluxos de processos, não dão margem para alterações, a menos que se reprograma todo o sistema. Assim, cada produto que requeira uma característica diferente na sua produção requer uma nova programação no seu fluxo de processo, o que implica em reprogramação de máquinas, ajuste de leiaute, programação dos transportadores, entre outros. A Figura 1 mostra o produto requerendo diversidade na produção. 
Figura 1: Necessidades de diversidade do produto.

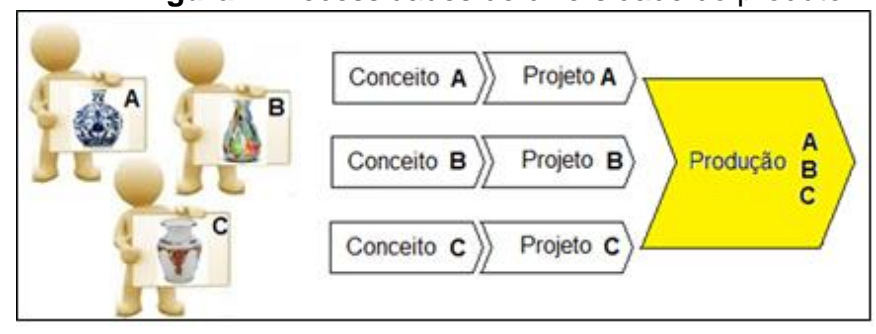

Fonte: Autor (2018).

Os segmentos de mercado que dependem diretamente do consumidor final estão susceptíveis a esta necessidade, o que deve ser encarado com uma oportunidade, pois trata-se de um consumidor que quer pagar para ter um produto diversificado. E para atender a este consumidor é necessário que os sistemas de produção possam serem flexíveis e adaptáveis, a ponto de se modificar rapidamente para atender a esta nova produção. Setores com o coureiro-calçadista, vestuário, mobiliário e automotivo, são exemplos de mercados em que os consumidores primam por ter produtos diferenciados e por vezes customizados.

Conceitos como Lean Manufacturing (SHINGO, 1996) e Just in time (JÔZEFOWSKA, 2007) objetivam a redução do tempo decorrido entre o pedido de um cliente e o envio do produto, através da eliminação de resíduos, redução do tempo de projeto, menores estoques, menos defeitos, redução dos setups, o que dão conta de lotes pequenos de fabricação, mas não dão conta de atender a diversidade. Os conceitos de Flexible Manufacturing System (KUSIAK, 1986), Compute Integrated Manufacturing e Agile Manufacturing (QUINN, 1997) trazem um certo dinamismo ao processo produtivo, mas não dão conta de modificar o fluxo produtivo para atender a diversidade requisitada.

O uso de sistemas multiagentes (BELLIFEMINE; GREENWOOD, 2007) como meio de interação entre os componentes do sistema de manufatura, se apresenta como uma solução hipotética ao problema da necessidade de interação dos componentes da manufatura, propiciando a negociação entre os recursos e a peça, com vistas a definir o fluxo de processos a medida da necessidade. $O$ sistema pode se auto-organizar, considerando os recursos que estão disponíveis no momento da requisição do serviço pela peça. A Figura 2 apresenta a proposta de solução com base na interação por agentes, para formar o conceito de auto-organização.

Figura 2: Hipótese com uso de agentes promovendo a interação.

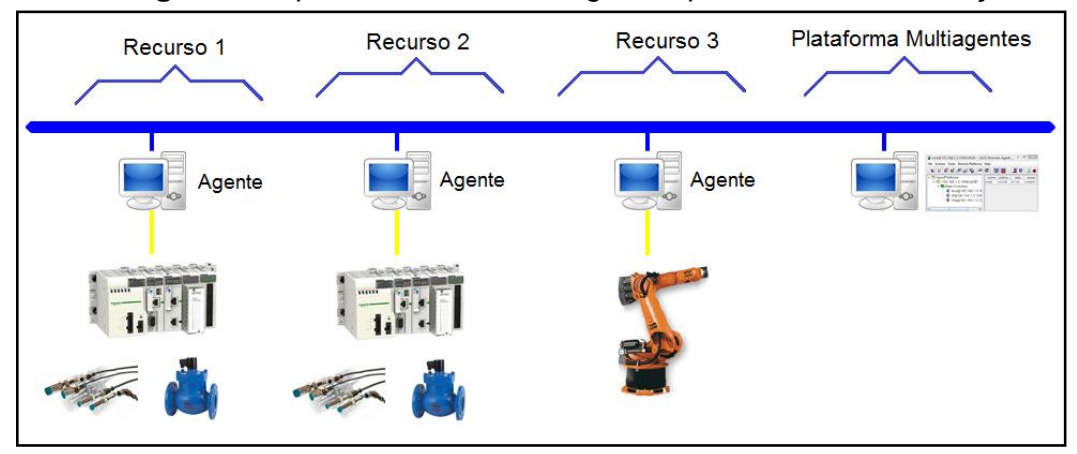

Fonte: Autor (2018). 


\section{Material e Métodos}

O conceito de CIM (Computer Integrated Manufacturing - Manufatura Integrada por Computador) é mais amplo e visa à integração entre todas as etapas do processo: vendas, suprimentos, projeto e desenvolvimento, produção, expedição e pós-vendas. Segundo (LEITÃO et al., 2001), o paradigma CIM consiste na integração de todas as atividades da empresa por meio da utilização das tecnologias da informação, como: bancos de dados, redes, aplicativos, entre outros, que permitem a troca e o compartilhamento de dados entre as unidades da empresa e suas aplicações. Assim, segundo (SCHEER, 2012), um CIM é composto pelos módulos seguintes: Projeto Assistido por Computador (CAD - Computer Aided Design); Planejamento Assistido por Computador (CAPP - Computer Aided Process Planning); Manufatura Assistida por Computador (CAM - Computer Aided Manufacturing); Controle de Qualidade Assistida por Computador (CAQ - Computer Aided Quality); Controle de Produção e Planejamento (PPC - Production Planning and Control).

O Planejamento de recurso corporativo - ERP - (SLACK; CHAMBERS; JOHNSTON, 2009) é um sistema de informação que integra todos os dados e processos de uma organização em um único sistema. A integração pode ser vista sob a perspectiva funcional (sistemas de finanças, contabilidade, recursos humanos, fabricação, marketing, vendas, compras, entre outros) e sob a perspectiva sistêmica (sistema de processamento de transações, sistemas de informações gerenciais, sistemas de apoio a decisão, entre outros). A proposta de um ERP é que todos os recursos que a produção de um determinado produto requeira sejam planejados de forma a serem requisitados de forma escalonada. Além da disposição do recurso, o planejamento de produto também obtém informações quanto ao custo de cada recurso, sua disposição e prazos de entrega. Assim, a manufatura de um produto pode sofrer modificações em seu processo em função da disponibilidade de recursos e obter minimização dos custos.

A partir de uma nova necessidade de mercado, com a redução de custos e melhor otimização dos recursos, surge então uma abordagem chamada Sistema de Gerenciamento da Produção (Manufacturing Execution Systems - MES), para os sistemas de automação da produção (McCLELLAN, 1997). A premissa se resume a proporcionar a troca de informações a partir dos sistemas de chão de fábrica com os sistemas de planejamento de negócios e fornecer aplicativos integrados em tempo real de produção. O termo MES foi originalmente criado em 1990 pela AMR - (Advance Manufacturing Research), uma empresa de pesquisas para a área industrial. Este primeiro conceito caracterizou o MES como uma classe de sistemas de informação que reside na camada de software que fica entre os sistemas de automação no chão de fábrica e os sistemas corporativos da companhia denominados ERP - (Entreprise Resource Planning). Um sistema MES é um sistema de controle para gerenciar e monitorar o processo de trabalho no chão de fábrica (QIU; ZHOU, 2016). Um MES mantém o controle de todas as informações de produção em tempo real, recebendo dados de robôs, máquinas e funcionários. O objetivo de um sistema MES é melhorar a produtividade e reduzir o tempo de ciclo, o tempo total para produzir uma ordem de produção. Ao integrar um MES com software ERP, os gerentes da fábrica podem ser proativos em garantir a entrega da produção com boa qualidade em tempo hábil e baixo custo. 
A Figura 3 apresenta a arquitetura relacional de um CIM, ERP e MES em um sistema produtivo.

Figura 3: Estrutura relacional de CIM, ERP e MES em um sistema produtivo.

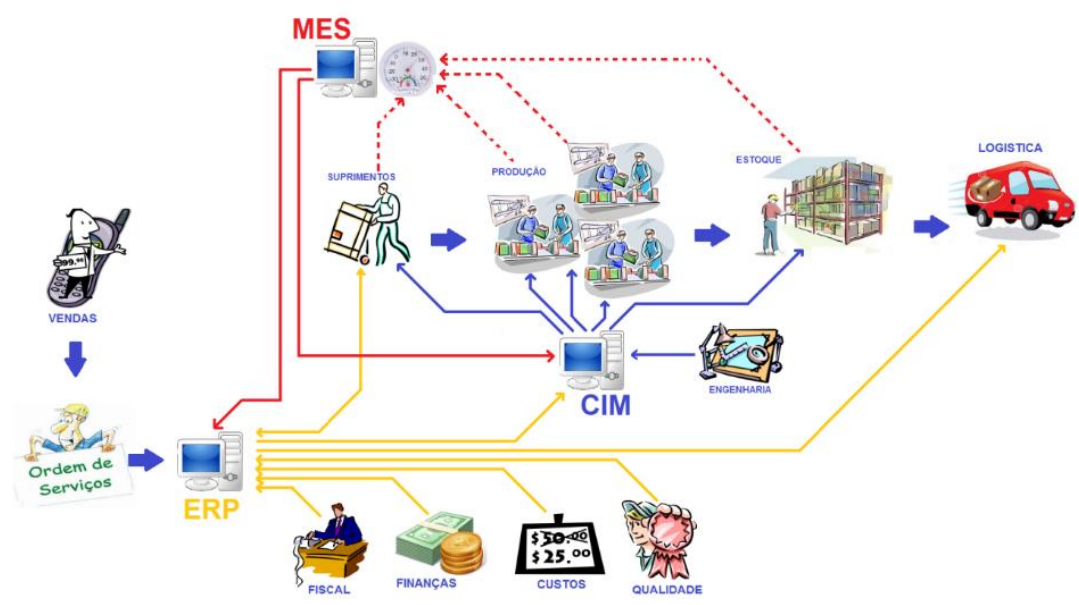

Fonte: Autor (2018).

Um sistema auto-organizável traz algumas vantagens aos sistemas produtivos. Com ele os elementos que afetam um sistema produtivo interagem entre si e buscam em conjunto a melhor solução para atender uma demanda de produção. Isto ocorre de forma dinâmica, a cada produto a ser produzido, a cada etapa da produção, tornando as decisões e arranjos produtivos mais assertivos. Esta evolução visa atender a uma necessidade de rápidas respostas a novas demandas de mercado, onde sistemas com hierarquia normalmente não dão conta (ONORI; SEMERE; LINDBERG, 2011). A hierarquia é definida pela peça a ser produzida. Quando um produto requer uma manufatura, ele interage diretamente com os componentes da manufatura para então definir a melhor forma de ser produzido. A Figura 4 apresenta a estrutura de um sistema auto-organizável em um sistema produtivo.

Figura 4: Estrutura de um sistema auto-organizável em um sistema produtivo.

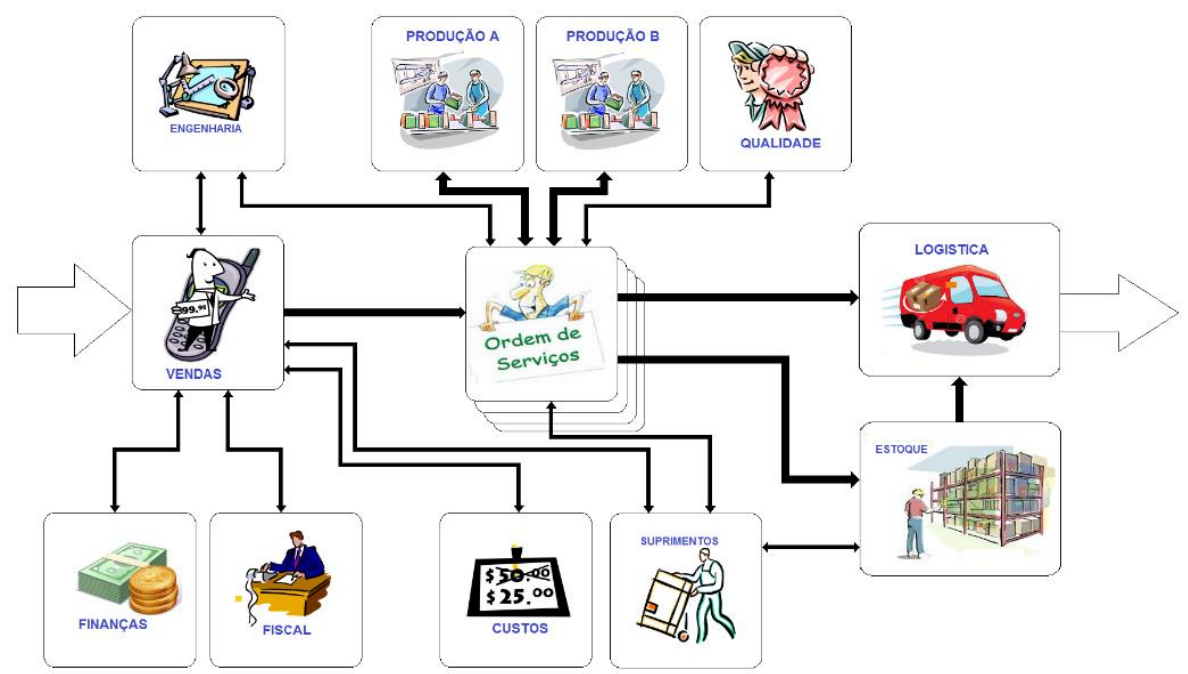

Fonte: Autor (2018). 
A Tabela 1 faz um comparativo entre os sistemas de manufatura industrial.

Tabela 1: Comparativo entre os sistemas de manufatura CIM, ERP, MES e Auto-organizável.

\begin{tabular}{|c|c|c|}
\hline $\begin{array}{l}\text { Sistema de } \\
\text { Manufatura } \\
\text { Industrial }\end{array}$ & Características importantes & Objetivo \\
\hline CIM & $\begin{array}{l}\text { Garante que o elemento } \\
\text { vinculado diretamente ao sistema } \\
\text { produtivo tenha os insumos e as } \\
\text { interconexões necessárias para } \\
\text { fabricar um dado produto. }\end{array}$ & $\begin{array}{l}\text { Orquestrar o fluxo de } \\
\text { produção. }\end{array}$ \\
\hline ERP & $\begin{array}{l}\text { Leva em consideração todos os } \\
\text { fatores que envolvem a } \\
\text { produção, não se } \\
\text { somente ao aspecto } \\
\text { mas também produtivo, } \\
\text { financeiros, custos, logística, } \\
\text { entre outros. }\end{array}$ & $\begin{array}{l}\text { Analisar completamente } \\
\text { os impactos e benefícios } \\
\text { na fabricação de um } \\
\text { produto desejado. }\end{array}$ \\
\hline MES & $\begin{array}{l}\text { Coleta dados dos tempos de } \\
\text { produção no meio produtivo, afim } \\
\text { de verificar o planejamento do } \\
\text { ERP. }\end{array}$ & $\begin{array}{l}\text { Fornecer informações } \\
\text { importantes do processo } \\
\text { para a tomada de } \\
\text { decisões em caso de } \\
\text { imprevistos em alguma } \\
\text { parte do processo. }\end{array}$ \\
\hline $\begin{array}{c}\text { Auto- } \\
\text { Organizável }\end{array}$ & $\begin{array}{l}\text { Promove a interação entre todos } \\
\text { os integrantes do sistema de } \\
\text { manufatura, a fim de alocar e } \\
\text { disponibilizar serviços. }\end{array}$ & $\begin{array}{l}\text { Gerenciar o fluxo } \\
\text { produtivo a partir do } \\
\text { produto, de forma } \\
\text { dinâmica, em tempo de } \\
\text { produção. }\end{array}$ \\
\hline
\end{tabular}

No trabalho de Peschl (2014) é abordada a importância de atender às mudanças rápidas das necessidades do consumidor, com o incremento do número de novos produtos e sua variedade. $\mathrm{A}$ manufatura do futuro terá que dar conta de uma variedade de produtos sendo produzidos na mesma linha de produção, assim como a flexibilidade de alterar a rota previamente estipulada. Com objetivo de desenvolver uma arquitetura de manufatura flexível de sistemas, que permite a fácil aceitação na indústria, baseia-se em uma abordagem de baixo para cima (botton up), com um novo tipo de componentes de chão de fábrica inteligentes e flexíveis, denominados "Manufactrons".

$\mathrm{Na}$ tese de doutoramento de Oliveira (2003) o autor aborda o problema da necessidade de agilidade no chão de fábrica, a fim de lidar com os distúrbios e incertezas que as empresas de fabricação enfrentam em seus cenários de negócios. A diversidade de produtos e a agilidade na produção e entregas fazem com que os dispositivos mecatrônicos existentes não consigam atender a estes novos requisitos. A capacidade de meios produtivos de serem melhorados rapidamente, alterados ou tornarem-se facilmente modificáveis dá ao meio produtivo a agilidade necessária para atender aos novos requisitos. Os elementos da manufatura são reunidos em coligações, constituídas de componentes de fabricação agentificado (módulos), cujas relações dentro das coalizões são regidas 
por contratos entre os elementos da coalizão, que estão configurados sempre que uma coalizão está estabelecida.

Já em Silva (2016), o trabalho é motivado pela necessidade de reconfiguração que os sistemas de manufatura de empresas demandam, de forma a atender diversidade de produtos através da reorganização do fluxo produtivo, auto-organizando os processos de produção.

O método proposto visa levar um sistema de manufatura a atuar de forma interativa. Proposto em Peixoto (2012), leva em consideração uma sequência de passos que devem serem executados, implementando o sistema multiagente que provocará a interação entre os elementos da manufatura. As etapas que compõe o método são:

a) 1a Etapa: identificação dos componentes que tem capacidade de funcionar autonomamente, mapeando-os como agente de recursos (RA);

b) 2a Etapa: verificar se os elementos de transporte devem ser mapeados como agentes de transporte (TUA);

c) 3atapa: considerar cada produto ou conjunto de produtos como um agente de produto (PA);

d) 4a Etapa: promover a implementação dos dispositivos como agentes, implementando em cada agente um módulo de interligação com o hardware, um módulo lógico que gerencie o dispositivo e um módulo de comunicação que possibilite a troca de mensagens com os demais agentes;

e) 5 Etapa: interligar os agentes em uma plataforma de comunicação entre agentes.

A Figura 5 apresenta a proposta do método, onde um sistema de manufatura é conduzido a um sistema interativo de manufatura, e a forma com que os agentes se interagem, negociando recursos e alocando tarefas.

Figura 5: Método para provocar a interação entre componente de uma manufatura industrial.

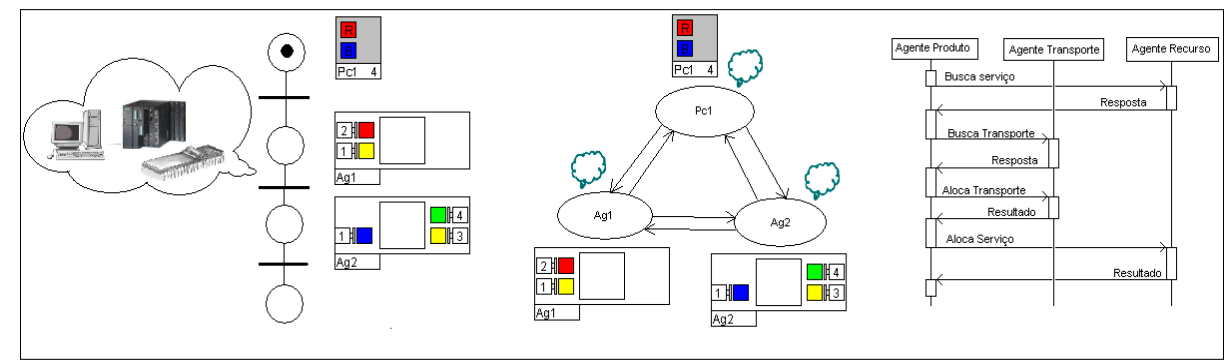

Fonte: Autor (2018).

Como forma de comparação entre os conceitos de manufatura propostos, uma planta de manufatura de processo contínuo é implementada, em um projeto conjunto com empresas vinculadas a ABIMAQ, onde os conceitos são ensaiados e métricas são observadas. A Figura 6 apresenta a planta de manufatura proposta e seus elementos. 
Figura 6: Estrutura de manufatura onde o método será aplicado.

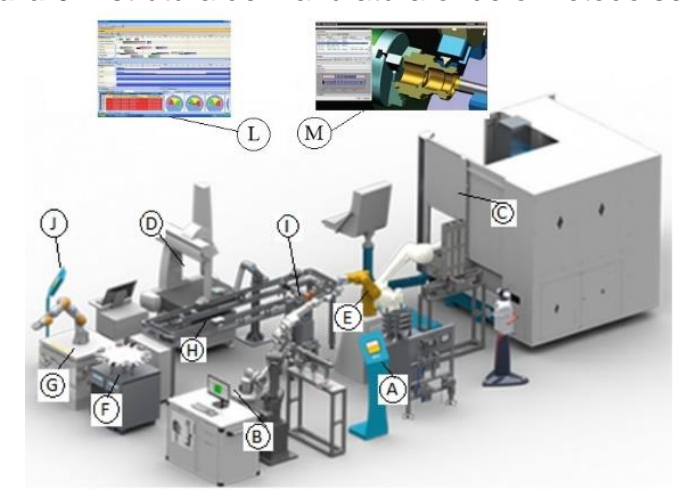

Fonte: Autor (2018).

O detalhamento de cada recurso na manufatura é descrito como: A - Toten de Entrada; B Escaneamento; C - Usinagem; D - Inspeção; E - Montagem; F- Almoxarifado; G - Entrega; H- Esteira; I - Descarte; J - Toten Saída; L - Gestão manufatura; M - Modelagem CNC. Cada componente da manufatura é responsável por uma etapa do processo fabril.

A intenção do artigo é propor um sistema de gestão da produção que dê conta das necessidades de diversidade dos produtos, premissa da Indústria 4.0. Nesta intenção, métricas que condizem com a necessidade do produto são definidas, como forma de qualificar os conceitos produtivos em questão. A Tabela 2 apresenta as métricas definidas, que serão observadas nos conceitos de manufatura ensaiados.

Tabela 2: Métricas a serem observadas nos conceitos de manufatura.

\begin{tabular}{|c|c|}
\hline Métricas [requisito] & Critério para avaliação \\
\hline $\begin{array}{l}\text { Manter a produção com a } \\
\text { mudança nos requisitos. }\end{array}$ & $\begin{array}{l}\text { O sistema deverá manter a produção sem } \\
\text { necessitar parar o sistema pela mudança de } \\
\text { requisitos do produto. }\end{array}$ \\
\hline $\begin{array}{l}\text { Manter a produção com } \\
\text { inserção de um único } \\
\text { produto em meio a um lote. }\end{array}$ & $\begin{array}{c}\text { A produção deve se manter mesmo com a } \\
\text { necessidade de fazer um único produto, } \\
\text { diferente do produto que se estava } \\
\text { produzindo. }\end{array}$ \\
\hline $\begin{array}{l}\text { Manter a produção com a } \\
\text { retirada de estações. }\end{array}$ & $\begin{array}{l}\text { Sendo o sistema modular, saindo uma das } \\
\text { estações, outra deve assumir a tarefa, } \\
\text { admitindo ter a mesma funcionalidade, sem } \\
\text { requerer alteração na programação. }\end{array}$ \\
\hline $\begin{array}{l}\text { Mudar a ordem de } \\
\text { processo em função da não } \\
\text { disponibilidade do recurso. }\end{array}$ & $\begin{array}{c}\text { A peça deve ser capaz de modificar sua } \\
\text { sequência de produção em função da não } \\
\text { disponibilidade do recurso. }\end{array}$ \\
\hline $\begin{array}{l}\text { Manter a produção com a } \\
\text { inserção de estações. }\end{array}$ & $\begin{array}{c}\text { O sistema deve permitir expansão. A } \\
\text { inserção de uma estação não deve requerer } \\
\text { alterações na programação, nem demandar } \\
\text { uma parada do sistema. }\end{array}$ \\
\hline $\begin{array}{l}\text { Manter a produção mesmo } \\
\text { com a mudança da estação } \\
\text { de posição física na planta. }\end{array}$ & $\begin{array}{l}\text { O sistema deve manter a produção mesmo } \\
\text { com a mudança de posição da estação na } \\
\text { planta. }\end{array}$ \\
\hline
\end{tabular}




\section{Resultados e Discussão}

O protótipo de sistema de manufatura foi montado como demonstrador de conceitos de produção que atenda a diversidade do produto. Exposto na FEIMEC2016, uma exposição de máquinas, ferramentas e conceitos de fornecedores e indústrias do segmento metalmecânico. Nesta planta é produzido um produto, que consiste em uma peça suporte para canetas e telefone celular, confeccionado para o tamanho do celular do cliente, com as cores das canetas que o cliente optar e com uma mensagem gravada na peça, escrita pelo cliente. Um produto customizado e lote único a ser produzido.

Os custos de implantação, por se tratar de mudança no conceito de programação em software, com a utilização dos mesmos componentes de automação, houve uma equiparação no custo te implantação.A Figura 7 apresenta o sistema de manufatura industrial implementado.

Figura 7: Sistema de manufatura industrial piloto implantado e exposto na FEIMEC2016.

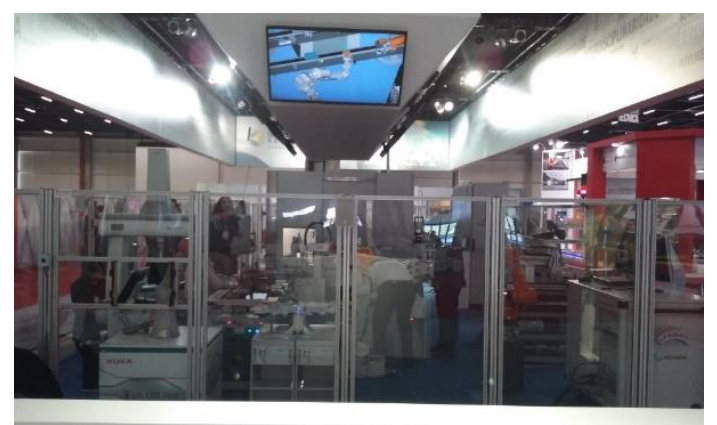

Fonte: Autor (2016).

Nesta proposta, inicialmente o conceito CIM foi implantado, em uma estrutura em que um controlador lógico programável central orquestra o fluxo produtivo a ser seguido. A Figura 8 apresenta o conceito CIM implementado.

Figura 8: Conceito CIM implementado na planta de manufatura piloto.

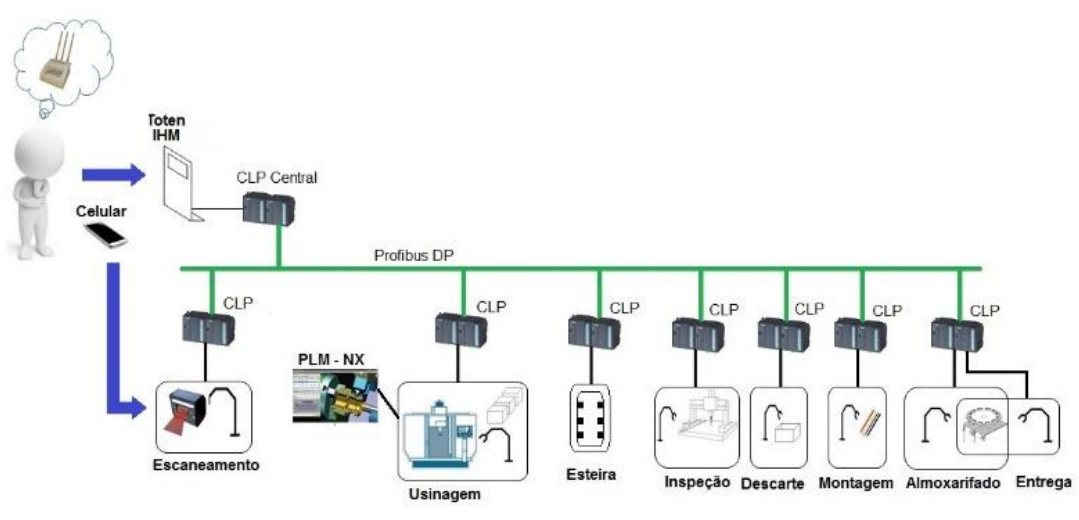

Fonte: Autor (2018). 
Após, o conceito MES foi implementado, em uma estrutura que o MES disponibilizava seus recursos à medida que ia sendo solicitado pela peça. A Figura 9 apresenta o conceito MES implementado.

Figura 9: Conceito MES implementado na planta de manufatura piloto.

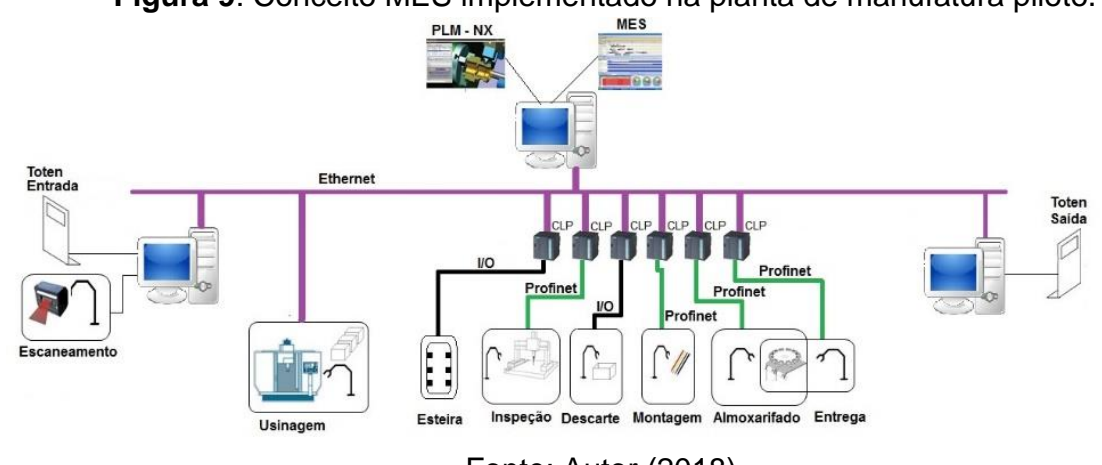

Fonte: Autor (2018).

Por fim, o sistema auto-organizável foi implementado, em uma estrutura que os controladores foram modelados como agentes de manufatura e interagiram entre si para propor e dispor serviços. $\mathrm{A}$ Figura 10 apresenta o conceito auto-organizável (self- $x$ ) implementado.

Figura 10: Conceito auto-organizável implementado na planta de manufatura piloto.

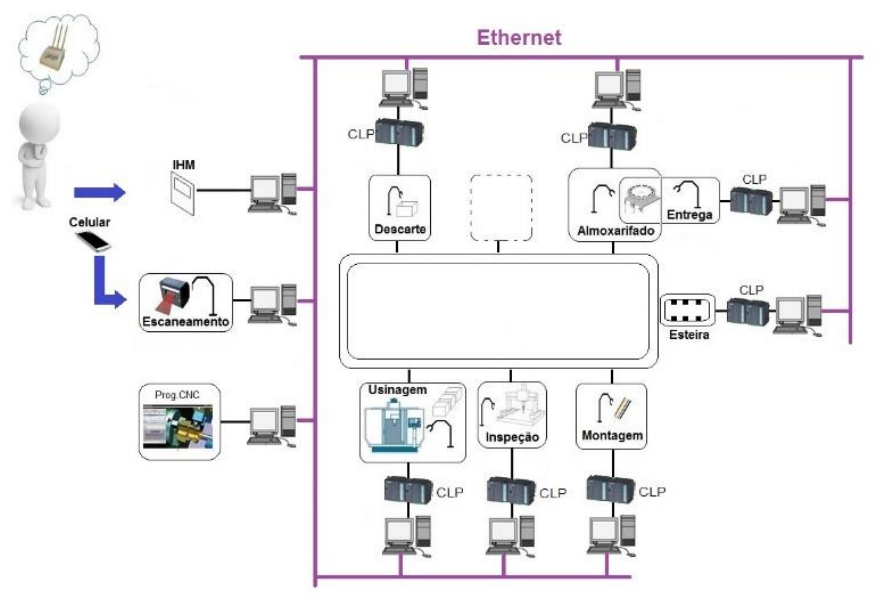

Fonte: Autor (2018).

Com os ensaios dos conceitos realizados, cada métrica foi medido e qualificado, sendo os resultados constantes na Tabela 3. Pela tabela apresentada e os resultados obtidos se observa que em comparação com o sistema gerenciado por CIM, MES e Auto-organizável (Self-x), os Sistemas Auto-organizáveis não requerem a parada, desligamento ou reprogramação do sistema caso haja necessidade de alterar os requisitos do produto, inserir novas estações, retirar estações ou modificar sua posição no sistema de manufatura. A essência do controle de cada estação continuou a mesma, pois continuaram com um controlador lógico programável e seu programa de controle não foi modificado, mas a ele foram agregadas as características de Sistemas Multiagentes com aplicação da metodologia proposta, o que levou o sistema de manufatura atender a diversidade, ser adaptável e ser Rev. Elet. Cient. UERGS, v.4, n.3, p. 525-538, 2018. 
auto-organizado, uma vez que o fluxo do processo produtivo se encontra descrito no agente que modela a peça e este é que determina a ordem com que os recursos serão utilizados. Os resultados mostram que o método é aplicável e leva o sistema com controlador lógico programável a atender às necessidades de diversidade, agilidade e auto-organização do meio produtivo.

Tabela 3: Resultados obtidos na utilização dos conceitos CIM, MES e Auto-organizável.

\begin{tabular}{|c|c|c|c|}
\hline Métricas & CIM & MES & Self-x \\
\hline $\begin{array}{c}\text { Manter a produção com a mudança } \\
\text { nos requisitos. }\end{array}$ & $\begin{array}{l}\text { Não } \\
\text { atende }\end{array}$ & Atende & Atende \\
\hline $\begin{array}{l}\text { Manter a produção com inserção de } \\
\text { um único produto em meio a um lote. }\end{array}$ & Atende & Atende & Atende \\
\hline $\begin{array}{c}\text { Manter a produção com a retirada de } \\
\text { estações. }\end{array}$ & $\begin{array}{l}\text { Não } \\
\text { atende }\end{array}$ & $\begin{array}{l}\text { Não } \\
\text { atende }\end{array}$ & Atende \\
\hline $\begin{array}{l}\text { Mudar a ordem de processo em } \\
\text { função da não disponibilidade do } \\
\text { recurso. }\end{array}$ & $\begin{array}{l}\text { Não } \\
\text { atende }\end{array}$ & Atende & Atende \\
\hline $\begin{array}{c}\text { Manter a produção com a inserção } \\
\text { de estações. }\end{array}$ & $\begin{array}{l}\text { Não } \\
\text { atende }\end{array}$ & $\begin{array}{l}\text { Não } \\
\text { atende }\end{array}$ & Atende \\
\hline $\begin{array}{l}\text { Manter a produção mesmo com a } \\
\text { mudança da estação de posição } \\
\text { física na planta. }\end{array}$ & $\begin{array}{l}\text { Não } \\
\text { atende }\end{array}$ & $\begin{array}{l}\text { Não } \\
\text { atende }\end{array}$ & Atende \\
\hline
\end{tabular}

Fonte: Autor (2018).

Analisando as métricas propostas se percebe que o conceito auto-organizável conseguiu ser eficiente em todas, atendendo o desejado para cada situação.

Dentre as métricas analisadas, destacam-se manter a produção com a mudança nos requisitos e mudar a ordem de processo em função da não disponibilidade do recurso. Os atendimentos a estas duas métricas indicam que os sistemas auto-organizáveis dão conta de atender à necessidade de diversidade do produto, pois o sistema produtivo é flexível e ágil suficiente para se modificar em seu fluxo de processo, para atender os novos requisitos, de forma dinâmica e sem a necessidade de parar o sistema produtivo para reprogramação.

Um aspecto a salientar é o fato de o controlador lógico ser modelado como um agente em um sistema multiagente, requer um maior conhecimento das pessoas que implementarão o sistema e das pessoas que irão operar o sistema, pois não se trata somente de uma aplicação de tecnologia, mas de uma mudança de conceito.

\section{Considerações finais}

Há uma tendência dos processos produtivos evoluírem para atuar de forma interativa, atendendo os preceitos da indústria 4.0, que aponta para virtualização dos processos, interoperabilidade, descentralização, adaptação da produção em tempo real, orientação a serviços e sistemas modulares dos equipamentos. E para isto é necessário que os recursos de um sistema 
produtivo tenham a capacidade de interação. Este artigo traz uma metodologia que leva o controlador lógico programável a ter a capacidade de interação, a partir das funcionalidades dos sistemas multiagentes que a ele fora disponibilizado. Com esta interação o trabalho leva os recursos de produção a interagirem entre si, ao encontro da tendência dos sistemas produtivos em atuarem conforme os preceitos da indústria 4.0, onde a auto-organização dá conta de atender os requisitos de diversidade do produto, desejado pelo mercado.

O produto customizado proposto foi atendido, face o caráter interativo que a planta apresentou, quanto implementou o conceito auto-organizável, sendo produzido a peça que se propunha a um suporte para telefone celular, com características próprias, customizadas pelo cliente.

A metodologia proposta visa o atendimento a processos em sistemas produtivos discretos. Isto não significa que a metodologia não se aplique a processos contínuo, porém, em uma análise prévia a aplicação do conceito de auto-organização faz mais sentido quando há concorrência entre recursos, e os processos contínuos já possuem um fluxo fisicamente definido, havendo poucos desvios de fluxo de seu processo. Mas cabe um estudo mais aprofundado para verificar a pertinência de aplicação da metodologia também nos processos contínuos.

\section{Referências}

BELLIFEMINE, F.; CAIRE, G.; GREENWOOD, D. Developing multi-agent systems with JADE. Chichester: John Wiley \& Son, 2007. (Wiley series in agent technology).

EUROPEAN PARLIAMENT. Industry 4.0: Digitalisation for productivity and growth. 2015. Disponível em:

<http://www.europarl.europa.eu/RegData/etudes/BRIE/2015/568337/EPRS_BRI(2015)568337_EN.pdf >. Acesso em: 29 fev. 2018.

HERMANN, M; PENTEK, T; OTTO, B. Design Principles for Industrie 4.0 Scenarios: a literature review. 2015. Disponível em: $<$ http://www.snom.mb.tudortmund.de/cms/de/forschung/Arbeitsberichte/Design-Principles-forIndustrie-4_0-Scenarios.pdf>. Acesso em: 29 fev. 2018.

JÔZEFOWSKA, J. Just-in-Time Scheduling: Models and Algorithms for Computer and Manufacturing Systems. Norwell: LLC, 2007. 255 p.

KUSIAK, A. Modelling and design of flexible manufacturing systems. Amsterdam: Northholland, 1986. $431 \mathrm{p}$.

LEITÃO, P. et al. Trends in agile and cooperative manufacturing. In: PROCEEDINGS OF THE LOW COST AUTOMATION SYMPOSIUM, 2001. Berlin. Proceedings... Berlin: Elsevier, 2001. Disponível em: <https://bibliotecadigital.ipb.pt/handle/10198/1426>. Acesso em: 12 abr. 2018.

McCLELLAN, M. Applying Manufacturing Execution Systems. Boca Raton: St. Lucie Press, 1997.

MENDES, J. M. et al. Service-Oriented Control Architecture for Reconfigurable Production Systems. In: IEEE INTERNATIONAL CONFERENCE ON INDUSTRIAL INFORMATICS -INDIN2008, 2008, Daejeon. Proceedings... New York: IEEE, 2008. Disponível em: <http://repositorioaberto.up.pt/bitstream/10216/25257/2/27473.pdf>. Acesso em: 9 feveireiro 2018.

OLIVEIRA, J. A. B. de. Coalition Based Approach for Shop Floor Agility: A Multiagent Approach. 2003. 329 f. Tese (Doutorado) - Curso de Engenharia Electrotécnica e de Computadores - Robótica e Manufactura Integrada, Faculdade de Ciências da Computação, Engenharia Elétrica e Matemática, 
Universidade Nova de Lisboa Uninova, Lisboa, 2003. Disponível em: <https://run.unl.pt/bitstream/10362/2483/1/Oliveira_2004.pdf>. Acesso em: 6 jan. 2018.

ONORI, M.; SEMERE, D.; LINDBERG, B. Evolvable systems: an approach to self-X production. International Journal of Computer Integrated Manufacturing, London, v. 24, n. 5, p.506516, May 2011. Informa UK Limited. Disponível em <http://dx.doi.org/10.1080/0951192x.2011.566282>. Acesso em mar 2018.

PEIXOTO, J. A. Desenvolvimento de sistemas de automação da manufatura usando arquiteturas orientadas a serviço e sistemas multiagentes. 2012. 134 f. Dissertação (Mestrado) - Curso de Pósgraduação em Engenharia Elétrica, Departamento de Engenharia Elétrica, Universidade Federal do Rio Grande do Sul, Porto Alegre, 2012. Disponível em: <http://hdl.handle.net/10183/61385>. Acesso em: 13 fev. 2018.

PESCHL, M. An architecture for flexible manufacturing systems based on taskdriven agents. 2014. 192 f. Tese (Doutorado) - Curso de Faculdade de Tecnologia da Informação e Engenharia Elétrica, Departamento de Ciências da Computação e Engenharia, Universidade de Oulu, Oulu, 2014. Disponível em: <http://herkules.oulu.fi/isbn9789526203669/isbn9789526203669.pdf>. Acesso em: 20 out. 2017.

QUINN, R. D. et al. An agile manufacturing workcell design. IIE Transactions. [S. I.], v. 29, n.10, 1997, p. 901-909. 1997. Disponível em <http://www.tandfonline.com/doi/abs/10.1080/07408179708966410>. Acesso em 12 de abr. 2018.

QIU, R. G.; ZHOU, M. Mighty MESs: State of the art and future manufacturing execution systems. IEEE Robotics \& Automation Magazine, New York, v. 11, n. 1, p.19-40, May. 2004. Disponível em: < http://dx.doi.org/10.1109/mra.2004.1275947>. Acesso em 6 de março de 2018.

SCHEER, A. W. CIM Computer Integrated Manufacturing: Towards the Factory of the Future. 3. ed. Dordrecht: Springer Science \& Business Media, 2012.

SHINGO, S. O sistema toyota de produção: do ponto de vista da engenharia de produção. Porto Alegre: Bookman, 1996. 291 p.

SLACK, N.; CHAMBERS, S.; JOHNSTON, R. Administração da Produção. 3. ed. São Paulo: Atlas, 2009.

SILVA, R. M. da. Controle de sistemas reconfiguráveis de manufatura. 2016. $157 \mathrm{f}$. Tese (Doutorado) - Curso de Ciências, Escola Politécnica, Universidade de São Paulo, São Paulo, 2016. 